\title{
Catalogue of type specimens of the Collection of Invertebrates of Instituto Nacional de Pesquisas da Amazônia, Manaus, Brazil. III. Hexapoda: Isoptera, Mantodea, Mecoptera, Orthoptera, Plecoptera, Trichoptera and Zoraptera
}

\author{
José Moacir Ferreira Ribeiro; Célio Magalhães ${ }^{2,3}$; José Albertino Rafael ${ }^{2,3}$ \& Augusto Loureiro Henriques ${ }^{2}$
}

\author{
${ }^{1}$ Instituto Nacional de Pesquisas da Amazônia, Programa PCI (MCT-INPA/CNPq), Caixa Postal 478, 69.011-970 Manaus, Brazil. \\ moacir@inpa.gov.br \\ ${ }^{2}$ Instituto Nacional de Pesquisas da Amazônia, celiomag, jarafael, loureiro@inpa.gov.br \\ ${ }^{3}$ Research fellow of the CNPq
}

\begin{abstract}
Catalogue of type specimens of the Collection of Invertebrates of Instituto Nacional de Pesquisas da Amazônia, Manaus, Brazil. III. Hexapoda: Isoptera, Mantodea, Mecoptera, Orthoptera, Plecoptera, Trichoptera and Zoraptera. A catalogue of the type specimens of Isoptera, Mantodea, Mecoptera, Orthoptera, Plecoptera, Trichoptera, and Zoraptera deposited in the Invertebrate Collection of the Instituto Nacional de Pesquisas da Amazônia (INPA), Manaus, Brazil, is presented and updated to December, 2007. A total of eight holotypes and seven lots of paratypes of nine species of Isoptera; three holotypes and one paratype of three species of Mantodea; five holotypes and five lots of paratypes of five species of Mecoptera; eight holotypes and five lots of paratypes of eleven species of Orthoptera; three holotypes, three neotypes and two lots of paratypes of seven species of Plecoptera; six holotypes and seven lots of paratypes of ten species of Trichoptera; and two holotypes and three lots of paratypes of three species of Zoraptera, are listed. Specific names are listed alphabetically within the family, followed by bibliographic citation, original genus name, status of type, collection number, locality data and remarks when appropriate.
\end{abstract}

KEYWORDS. Amazon Region; Arthropoda; INPA; taxonomy; type collection.

RESUMO. Catálogo dos espécimes-tipo de invertebrados da coleção do Instituto Nacional de Pesquisas da Amazônia, Manaus, Brasil. III. Hexapoda: Isoptera, Mantodea, Mecoptera, Orthoptera, Plecoptera, Trichoptera e Zoraptera. É apresentado o catálogo dos espécimes-tipo de Isoptera, Mantodea, Orthoptera, Plecoptera, Trichoptera e Zoraptera depositados na Coleção de Invertebrados do Instituto Nacional de Pesquisas da Amazônia (INPA), Manaus, Brasil, atualizado até dezembro de 2007. É relacionado um total de sete holótipos e sete lotes de parátipos de nove espécies de Isoptera; três holótipos e um parátipo de três espécies de Mantodea; cinco holótipos e cinco lotes de parátipos de cinco espécies de Mecoptera; oito holótipos e cinco lotes de parátipos de 11 espécies de Orthoptera; três holótipos, três neótipos e dois lotes de parátipos de sete espécies de Plecoptera; seis holótipos e sete lotes de parátipos de dez espécies de Trichoptera; e dois holótipos e três lotes de parátipos de três espécies de Zoraptera. São listados alfabeticamente em cada família os nomes das espécies, seguidos da citação bibliográfica, nome original do gênero, categoria do tipo, número de registro na coleção, dados de localidade e comentários quando pertinentes.

PALAVRAS-CHAVE. Arthropoda; Região Amazônica; INPA; taxonomia; coleção de tipos.

The Invertebrate Collection of the Instituto Nacional de Pesquisas da Amazônia (INPA) was initiated in 1954 and, by the end of 2007, housed type material of more than 900 species. Almost $80 \%$ of this amount belong to various Hexapoda groups. A numbered series of catalogues of its type material was planned to cover different taxa, given that the preparation of a single, complete volume is not feasible within a short period of time. The first part I, (Magalhães \& Pimpão 2006) comprises Acanthocephala, Nematoda, and Rotifera; The second part II list the types of Collembola, Diplura, Archaeognatha, Ephemeroptera and Blattaria (Ribeiro et al. 2008). This paper continues, as part III, listing type material of Isoptera, Mantodea, Mecoptera, Orthoptera, Plecoptera, Trichoptera, and Zoraptera.

\section{MATERIALAND METHODS}

The present catalogue follows the same format and procedures established by Magalhães \& Pimpão (2006) and Ribeiro et al. (2008).

\section{RESULTS}

A total of eight holotypes and seven lots of paratypes of nine species of Isoptera; three holotypes and one paratype of three species of Mantodea; five holotypes and five lots of paratypes of five species of Mecoptera; eight holotypes and five lots of paratypes of 12 species of Orhoptera; three holotypes, three neotypes and two lots of paratypes of seven species of Plecoptera; six holotypes and seven lots of 
paratypes of ten species of Trichoptera and two holotypes and three lots of paratypes of three species of Zoraptera were found to be currently deposited at the INPA's entomological collection.

\section{LIST OFTYPE SPECIMENS}

\section{Order ISOPTERA \\ KALOTERMITIDAE}

breviceps Constantino, 1997: 126, figs 1-8. Eucryptotermes

Holotype: male, ISOPT 001, alcohol. [Brazil, Amazonas, Balbina, 16.iv.1984, Leg. F. Campos]. Paratypes: 7 spec., ISOPT 001, one vial with alcohol, three soldiers and four nymphs; data as holotype.

\section{TERMITIDAE}

ayri Bandeira \& Cancello, 1992: 432, figs 14-16. Termis

Holotype: ISOPT 003, alcohol. [Brazil], Roraima, ilha de Maracá, 26.x.1987, Leg. S.P. Staide. Paratypes: 15 spec., ISOPT 003, two vials with alcohol, one with nine specimens and one with six specimens; locality data as holotype.

caapora Bandeira \& Cancello, 1992: 424, figs 1-6.

Paracornitermis

Holotype: male, ISOPT O07, alcohol. [Brazil], Roraima, ilha de Maracá, 22.x.1987, Leg. L. Antony and A.G. Bandeira.

crassilabrum Constantino, 1995: 478, figs 113-120.

\section{Syntermes}

Holotype: male, ISOPT 004, alcohol. Brazil, Amazonas, Manaus, Reserva [Florestal Adolpho] Ducke, 23.i.1991, Leg. F.B. Apolinário. Paratypes: 2 spec., ISOPT 004, one vial with alcohol; data as holotype.

cupiporanga Bandeira \& Cancello, 1992: 430, figs 11-13.

Dentispicotermes

Holotype: male, ISOPT 005, alcohol. [Brazil], Roraima, ilha de Maracá, 04.ii.1988, Leg. L. Antony and A.G. Bandeira. Paratypes: 65 spec., ISOPT 005, one vial with alcohol; data as holotype.

longilabrum Constantino, 1998: 111, figs 1-7.

Planicapritermes

Holotype: ISOPT 002, alcohol. [Brazil, Amazonas], Balbina, 16.iv.1996, Leg. F. Campos. Paratypes: 4 spec., ISOPT 002, one vial with alcohol; data as holotype.

triceratops Cancello \& Bandeira, 1992: 3, figs 1-8.

\section{Macuxitermes}

Holotype: male, ISOPT 008, alcohol. [Brazil], Roraima, ilha de Maracá, 11.ii.1988, Leg. A.G. Bandeira.

mirim Bandeira \& Cancello, 1992: 428, figs 7-10.

Neocapritermes

Holotype: male, ISOPT 006, alcohol. [Brasil], Roraima, ilha de Maracá, 16.ii.1988, Leg. L. Antony and A.G. Bandeira. Paratypes: 38 spec., ISOPT 006, three vials with alcohol; one with 23 specimens, one with seven specimens and one with eight specimens.

\section{SERRITERMITIDAE}

sulcatus Cancello \& DeSouza, 2004: 5, figs 1-12. Glossotermes

Paratypes: 35 spec., ISOPT 009, one vial with alcohol. [Brazil], Amazonas, Manaus, Estação Experimental de Silvicultura Tropical, Manaus-Boa Vista (BR-174) road, Km 44, 05.iv.1992, Leg. A.G. Bandeira.

\section{Order MANTODEA \\ MANTIDAE}

amazonica Jantsh, 1985: 1. Urumantis

Holotype: male, MANTI 001. Brazil, Amazonas, Itacoatiara, estrada AM 010, Km 232, 17.xii.1977, Leg W.E. Kerr. Paratype: male, MANTI 001. Brasil, Amazonas, Itacoatiara, 02.ix.1977, Leg. Rufino.

paraensis Jantsh, 1985: 1. Urumantis

Holotype: male, MANTI 002. Brazil, Pará, Uruará , 65 Km SW Itaituba on BR 230 road, 12-15.x.1977, Leg. B.C. Ratcliffe.

\section{HYMENOPODIDAE}

corseuili Jantsh, 1986: 1. Tithrone

Holotype: male, MANTI 003. United Sates of America, California, 07.vii.1972, unknown collector.

\section{Order ORTHOPTERA ACRIDIDAE}

manausana Decamps, 1981: 320, figs 24-30. Anablysis

Holotype: male ORTHO 018. [Brasil], Amazonas, Reserva Biológica do Cuieiras, $50 \mathrm{Km} \mathrm{N}$, 1979, unknown collector. Paratype: female ORTHO 019 (Alotype); data as holotype.

punctipes Decamps, 1981: 323, figs 40, 48, 56, 61, 62. Aptoceras

Holotype: male ORTHO 016. [Brasil], Amazonas, Reserva Biológica do Cuieiras, $50 \mathrm{Km} \mathrm{N}$, 1979, unknown collector.

rubriventris Decamps, 1981: 327, figs 65-x71. Miacris

Holotype: male, ORTHO 017. [Brasil], Amazonas, Reserva Biológica do Cuieiras, $50 \mathrm{Km} \mathrm{N}$, 1979, unknown collector.

\section{EUMASTACIDAE}

dukeana Decamps, 1982: 169, figs 111-114. Eumastacops

Holotype: male, ORTHO 003, [Brasil, Amazonas] Reserva [Florestal Adolpho] Ducke, v.1963, Leg. Faustino. Paratype: male, ORTHO 004; data as in holotype.

sinopensis Decamps, 1982: 177, figs 88, 131. Pseudomastacops

Paratypes: 2 males, ORTHO 005 and 006. Brasil, Mato Grosso, Aripuanã, Reserva Humboldt (10 $10^{\circ}$ 'S $\left./ 59^{\circ} 48^{\prime} \mathrm{W}\right), 23 . i .1976$ and 16-22.iii.1977, Legs. A.P.A. Luna Dias and B.C. Ratcliffe.

uruana Decamps, 1982: 177, figs 88, 131. Pseudomastacops

Holotype: male, ORTHO 007. Brasil, Pará, Urua, 65 km SW Itaituba, on BR- 230, 12-15.x.1977, Leg B.C. Ratcliffe.

\section{ROMALEIDAE}

cantans Decamps, 1981: 316, figs 9-11. Prionacris

Holotype: male, ORTHO 015. [Brasil], Amazonas Reserva Biológica do Cuieiras, 1979, unknown collector. corticolor Decamps, 1978: 321, figs 62-63. Hylephilacris

Holotype: male, ORTHO 001. [Brasil, Amazonas], Manaus, [campus] INPA-sede, 15.iv.1976, Leg. A.P.A. Luna Dias. gloriosa Decamps, 1981: 312. Ophthalmolampis

Holotype: male, ORTHO 008. [Brasil], Amazonas, Reserva Biológica do Cuieiras, $50 \mathrm{Km} \mathrm{N}$, [15.ix and 30.x.]. 1979, unknown collector. Paratypes: 2 spec., ORTHO 009 (allotype) and 010 .

magnicornis Decamps, 1978: 320, figs 52-61. Hylephilacris

Paratype: 1 spec., male, ORTHO 002. [Guyana, Sinnamary], Km 2025, vii.1977, Leg. M. Decamps. 
nigricans Decamps, 1981: 311. Pseudonautia

Holotype: male, ORTHO 011. [Brasil], Amazonas, Reserva Biológica do Cuieiras, 1979, unknown collector. Paratypes: 3 spec., ORTHO 012, (allotype) ORTHO 013 and ORTHO 014, data as in holotype.

\section{Order MECOPTERA BITTACIDAE}

araguaiensis Penny \& Arias, 1983: 701, figs 1-3. Issikiella

Holotype: male, MECOP 017. Brasil, Pará, Conceição do Araguaia, 19-24.i.1983, Leg. N.D. Penny. Paratypes: 18 spec., nine male and nine female MECOP 018-035, one specimen being designated as allotype (018) locality data as holotype.

aripuanaensis Penny, 1977: 423. figs 1-4. Neobittacus

Holotype: male, MECOP 001. [Brasil]. Mato Grosso, Aripuaná, Reserva Humboldt, estrada da Colônia, 02.x.1975, Leg. L .P. Albuquerque et al. Paratypes: 2 spec., MECOP 002 (allotype) and MECOP 003, locality data as holotype.

byersi Penny \& Arias, 1982: 263, figs 1-4. Issikiella

Holotype: male, MECOP 007. Brasil, Amazonas, [Manaus], Reserva [Florestal Adolpho] Ducke, 26.xii.1979, Leg. M. Mendonça. Paratypes: 4 spec., females, MECOP 008 (allotype), 009 and 010, locality data as holotype.

ornaticaudus Penny, 1983: 424, figs 5-9. Pazius

Holotype: male, MECOP 004. Brasil, Mato Grosso, Reserva Humboldt, 21.ii.1978, Leg. N.D. Penny. Paratypes: 2 spec., females, MECOP 005 (allotype) and MECOP 006, locality data as holotype. souzalopezi Penny \& Arias, 1983: 266, figs 5-7.

Nannobittacus

Holotype: male, MECOP 011. Brasil, Amazonas, [Manaus], Reserva [Florestal Adolpho] Ducke, 21.iii.1977, Leg. J. Arias. Paratypes: 5 spec., females, MECOP 012 (allotype), MECOP 016-MECO 018, locality data as holotype.

\section{Order TRICHOPTERA HYDROPSYCHIDAE}

amazonense Flint, 1978: 399, figs 130-134,145. Leptonema

Holotype: male, TRICH 001. Brasil, Amazonas, Manaus, Reserva [Florestal Adolpho] Ducke, 24.xi.1976, Leg. N.D. Penny. amazonense Flint, 1978: 403, figs 142-144. Macronema

Holotype: male, TRICH 002. Brasil, Amazonas, Manaus, [Reserva Egler], Estrada AM 010, Km 64, 24.viii.1970, [Leg. A. Faustino]. exophthalmum Flint, 1978: 401, fig. 148. Macronema

Paratype: female, TRICH 003. Brasil, Amazonas, Manaus, Reserva da Campina, $60 \mathrm{Km}$, N of Manaus, 14.i.1977, Leg. N.D. Penny. gladiator Flint, 1978: 379, figs 33-35. Smicridea

Paratype: male, TRICH 005. Brasil, Amazonas, Manaus, Reserva [Florestal Adolpho] Ducke, 14.iii.1977, Leg. N.D. Penny.

hamadae Holzenthal \& Paes, 2004: 8, figs 1-9. Amazonatolica

Holotype: male, TRICH 011; Brazil, Amazonas, Manaus, Reserva Florestal Adolpho Ducke, 0257'S 5957'W, Igarapé Barro Branco (sede), vi.2001, Leg. J. Vidal. Paratypes: 92 spec., TRICH 011 (192), 12 vials with alcohol, one vial with 20 specimens, one vial with nine specimens, one vial with two specimens, two vials with two specimens, one vial with five specimens, one vial with 25 specimens, one vial with two specimens, two vial with eight specimens, one vial with five specimens and one vial with 13 specimens; different locality of holotype.

neotropicalis Flint, 1978: 70, figs 8-12. Tricholeiochiton

Holotype: male, TRICH 009. Brasil, Roraima, Maracá, Rio Uraricoera, 02-13.v.1987, Leg. J.A. Rafael et al. Paratypes: 21 spec., TRICH INPA 009 (1-21); data as holotype. pennyi Flint, 1978: 401, figs 139-141, 149. Macronema

Holotype: male, TRICH 004. Brasil, Amazonas, Manaus, Reserva [Florestal Adolpho] Ducke, 24.xi.1976, Leg. N.D. Penny.

rafaeli Flint, 1991: 74, figs 13-15. Oecetis

Holotype: male, TRICH 010. Brasil, Roraima, Ilha de Maracá, Rio Uraricoera, 18-28.viii.1987, Leg. J.A. Rafael et al. Paratypes: 4 spec., TRICH 010 (1-4); locality data as holotype.

rostratum Flint, McAlpine \& Ross 1987: 30, figs 195-202.

Leptonema

Paratypes: 1 spec., female, TRICH 008. Brasil, Roraima, Rio Mucajaí, 14.v.1977, Leg. N.D. Penny. 1 spec., female, TRICH 008; Mato Grosso, Reserva Humboldt, 12.iv.1979, Leg. N.D. Penny.

voluta Flint, 1978: 378, figs 27-29. Smicridea

Paratypes: 1 spec., males, TRICH 006 and TRICH 0071 spec., female. Brasil, Amazonas, Manaus, 08.iii.1977, Leg. N.D. Penny.

\section{Order ZORAPTERA ZOROTYPIDAE}

amazonensis Rafael \& Engel, 2006: 3, figs 1-14. Zorotypus

Holotype: male, ZORAP 003a, slide. Brasil, Amazonas, Itacoatiara Madeireira Mil, 17.iii.2002, Leg. J.A. Rafael and João Vidal. Paratypes: 5 spec., ZORAP 003b-f, slide, and 1 spec., alcohol; locality data as holotype.

weidneri New, 1978: 365, figs 7-15. Zorotypus

Holotype: male, ZORAP 001, slide. Brasil, Manaus, Reserva [Florestal Adolpho] Ducke, INPA, 12.xii.1975, Leg. H.D. Hamilton. Paratypes: 2 spec., ZORAP 001, slides; data as in holotype.

hamiltoni New, 1978: 365, figs 16-123. Zorotypus

Paratypes: 2 spec., 1 male and 1 female, ZORAP 002a-b, slides. Colombia, Purace, Maremberg, Huila, 30.ii.1973, Leg. H.D. Hamilton.

\section{Order PLECOPTERA PERLIDAE}

anae Ribeiro \& Rafael, 2007: 26, figs.35-43. Macrogynoplax Holotype: male, PLECO 003, alcohol (dissected abdomen in an Eppendorf vial). Brasil, Amazonas, Manaus, Reserva Florestal Adolpho Ducke, Igarapé Ipiranga, Malaise, 13-20.v.2003, leg. J.M.F.Ribeiro \& João Vidal. Paratypes: 2 spec., PLECO 003, two Eppendorf vials with alcohol, locality data as holotype

delicata Ribeiro-Ferreira \& Froehlich, 1999: 134, figs. 1-5.

Macrogynoplax

Neotype: (designation by Ribeiro \& Rafael, 2007), male, PLECO 005, Eppendorf vial with alcohol. Brasil, Amazonas, Manaus, Reserva Florestal Adolpho Ducke, Igarapé Bolívia, Malaise ,24-28.ii2003., Leg. J.M.F. Ribeiro.

froehlichi Ribeiro-Ferreira, 1996: 145, figs. 1-6. Enderleina

Holotype: male, PLECO 007, Eppendorf vial with alcohol. Brasil, Amazonas, Manaus, Reserva [Florestal Adolpho] Ducke, AM 010 Km 26, Igarapé Anta, 13.xi.1987, Leg. A.C. Ferreira.

manauensis Ribeiro-Ferreira in Ribeiro-Ferreira \& Froehlich,

2001: 189, figs 5-10. Anacroneuria

Holotype: male, PLECO 001, vial with alcohol and aedeagus in a micro vial with glycerin. Brasil, Amazonas, Manaus, Reserva [Florestal Adolpho] Ducke, Malaise, 01.i.1989, Leg. U.C. Barbosa. Paratypes: 3 females, PLECO 001 in two vials with alcohol, one with three specimens, one with legs and one aedeagus in micro vial with glycerin; locality data as holotype.

marlieri Froehlich in Ribeiro-Ferreira \& Froehlich, 2001:

189, figs 11-16. Anacroneuria

Neotype: (designation by Ribeiro \& Rafael, 2007, male, PLECO 002, Eppendorf vial with alcohol and aedeagus in a micro vial with 
glycerin. Brasil, Amazonas, Manaus, Reserva [Florestal Adolpho] Ducke, Igarapé Ipiranga, Malaise, 31.xi.2003, Leg. J.M.F. Ribeiro \& J. Vidal.

poranga Ribeiro-Ferreira \& Froehlich, 1999: 136, figs. 1418. Macrogynoplax

Neotype: (designation by Ribeiro \& Rafael, 2007), male, PLECO 006, Eppendorf vial with alcohol. Brasil, Amazonas, Manaus, Reserva [Florestal Adolpho] Ducke, Igarapé Tinga, Malaise trap, 2431.iii.2003, Leg. J.M.F. Ribeiro.

pulchra Ribeiro-Ferreira \& Froehlich, 1999: 134, figs. 7-13.

\section{Macrogynoplax}

Holotype: male, PLECO 004, specimen in a vial with alcohol, an Eppendorf vial with exuvia nimphal and a micro vial with glycerin with the aedeagus. Brasil, Amazonas, Manaus, Reserva [Florestal Adolpho] Ducke, Igarapé Barro Branco, 05.iii.1988, Leg. A.C. Ferreira.

Acknowledgments. J. M. F. R. thanks the Instituto Nacional de Pesquisas da Amazônia for scholarships received as part of the PCI Program (MCT-INPA/CNPq). C.M. and J.A.R. thanks CNPq for ongoing research grants. We are grateful to B. Robertson for correcting the English text.

\section{REFERENCES}

Bandeira, A. G. \& E. M. Cancello. 1992. Four new species of Termites (Isoptera, Termitidae) from the Island of Maracá, Roraima, Brazil. Revista Brasileira de Entomologia 35: 423-435.

Cancello, E. M. \& A. G. Bandeira. 1992. Macuxitermes triceratops (Isoptera: Termitidae: Nasutitermitinae), a new genus and species from Island of Maracá, Roraima. Papeis Avulsos de Zoologia 38: $1-8$.

Cancello \& E. M. DeSouza. 2004. A new species of Glossotermes (Isoptera): reappraisal of the generic status with transfer from the Rhinotermitidae to the Serritermitidae. Sociobiology 44: 1-19.

Constaino, R. 1998. Description of a new Planicapritermes from Central Amazonia, with notes on the morphology of the digestive tube of the Neocapritermes group (Isoptera: Termitidae: Termitinae). Sociobiology 32: 109-144.

Descamps, M. 1978. Ètude des ecosystèmes guyanais. III: Acridomorpha dendrophilos (Orthoptera-Caelifera). Annales de la Société. Entomologique de. France 14: 301-349.

Descamps, M. 1981. La faune dendrophile Néotropicale VI. Diagnoses génériques et spécifiques d'Acridoidea de la région de Manaus (Orthoptera). Annales de la Société Entomologique de France 17: $311-330$.

Descamps, M. 1982. Eumastacoidea néotropicaux, diagnoses, signalisations, notes biologiques. II. Bulletin de la Société Entomologique de France 87: 141.

Descamps, M. 1983. La Faune Dendrophile Néotropicale IX. Seconde revue des Ophthalmolampini. Le groupe des Ophthalmolampae (Orthoptera, Romaleidae). Annales de la Société.
Entomologique de France 19: 367-404.

Flint, O. S. 1978. Studies of Neotropical caddisflies. XXII. Hydropsychidae of the Amazon Basin (Trichoptera). Amazoniana 6: $373-421$.

Flint, O. S. 1991. Studies of Neotropical caddisflies. XLIV: A collection from ilha de Maraca, Brazil. Acta Amazonica 21: 63-83.

Froehlich, C. G. 2003. Anacroneuria (Plecoptera: Perlidae) from the Brazilian Amazonia with the description of three new species and a key to Macrogynoplax. Studies on Neotropical Fauna and Environment 38: 129-134.

Jantsh, L. J. 1985. Descrição de duas espécies de Louva-a-Deus Neotropicais (Mantodea, Mantidae, Mantinae, Mantini). Revista Brasileira de Entomologia 29: 315-316.

Jantsh, L. J. 1986. Descrições de duas novas espécies de Acantiothespini (Mantodea, Hymenopodidae, Acromantinae). Revista Brasileira de Entomologia 30: 13-15.

Magalhães, C. \& D. M. Pimpão. 2006. Catalogue of type specimens of invertebrates in the collection of the Instituto Nacional de Pesquisas da Amazônia, Manaus, Brazil. I. Acanthocephala, Nematoda, and Rotifera. Revista Brasileira de Zoologia 23: 630-636.

New, T. R. 1978. Notes on Neotropical Zoraptera, with descriptions of two new species. Systematic Entomology 3: 361-370.

Penny, N. D. \& J. R. Arias. 1982. Notes on Amazonian Bittacidae (Mecoptera) with the descriptions of two new species. Memorias do Instituto Oswaldo Cruz 77: 263-274.

Penny, N. D. \& J. R. Arias. 1983. A new species of Issikiella from the Amazon Basin (Mecoptera: Bittacidae). Acta Amazonica 13: 701704.

Penny, N. D. 1977. Two new species of Bittacidae (Mecoptera) from the Amazon Basin. Acta Amazonica 7: 423-427.

Rafael, J. A. \& M. S. Engel. 2006. A new species of Zorotypus from Central Amazonia, Brazil (Zoraptera: Zorotypidae). American Museum Novitates 35: 1-11.

Holzenthal, R. H. \& A. M. O. Pes. 2004. A new genus of long-horned caddisfly from the Amazon basin (Trichoptera: Leptoceridae: Grumichellini). Zootaxa 621: 1-16.

Ribeiro, J. M. F. \& J. A. Rafael. 2007. Description of one new species and a key to adults of Macrogynoplax Enderlein (Plecoptera, Perlidae) from Reserva Florestal Adolpho Ducke, Amazonas, Brazil. Zootaxa 1511: 17-28.

Ribeiro, J. M. R.; C. Magalhães; J. A. Rafael \& A. L. Henriques. 2008. Catalogue of type specimens of invertebrates in the collection of the Instituto Nacional de Pesquisas da Amazônia, Manaus, Brazil. II. Hexapoda: Collembola, Diplura, Archaeognatha, Ephemeroptera and Blattaria. Revista Brasileira de Entomologia 52: 595598.

Ribeiro-Ferreira, A. C. \& C. G. Froehlich. 2001. Anacroneuria Klapálek, 1909 from Amazonas State, North Brazil (Plecoptera, Perlidae, Acroneuriinae). Aquatic Insects 23: 187-192.

Ribeiro-Ferreira, A. C. \& C. G. Froehlich. 1999. New species of Macrogynoplax Enderlein, from North Brazil (Plecoptera, Perlidae, Acroneuriinae). Aquatic Insects 23: 133-147.

Ribeiro-Ferreira, A. C. 1996. Nova espécie de Enderleina Jewett do Norte do Brasil (Plecoptera: Perlidae). Acta Amazonica 25: 145147. [1996 (1995)]. 